\title{
Eletrocardiografia contínua (Holter) em cães saudáveis submetidos a diferentes exercícios físicos
}

\author{
[Ambulatory electrocardiography (Holter) in healthy dogs submitted to \\ different physical exercises] \\ F.L. Barretto ${ }^{1}$, F.S. Ferreira ${ }^{1}$, M.V. Freitas $^{2}$, V.S. Santos ${ }^{3}$, E.S. Correa ${ }^{1}$, C.B. Carvalho ${ }^{1}$ \\ ${ }^{1}$ Universidade Estadual do Norte Fluminense Darcy Ribeiro - Campos dos Goytacazes, RJ \\ ${ }^{2}$ Universidade Federal de Lavras - Lavras, MG \\ ${ }^{3}$ Médica veterinária autônoma
}

\begin{abstract}
RESUMO
O presente trabalho teve como objetivo avaliar os efeitos de diferentes cargas de exercícios físicos no comportamento da frequência e do ritmo cardíaco de cães saudáveis, ao longo das 24 horas de gravação Holter. Utilizaram-se 23 cães, sem restrição de sexo, idade ou raça, dos quais 15 foram submetidos a atividades físicas e pertenciam ao grupo controle. Os resultados mostraram alterações na frequência cardíaca durante as atividades propostas, em que se alcançaram $174 \pm 27 \mathrm{bpm}$ durante a caminhada leve, $148 \pm 30$ durante a atividade com bola e 165 \pm 26 durante o Cooper. Ademais, observaram-se arritmias, incluindo bloqueios atrioventriculares e extrassístoles e maior número e duração de pausas (até $5 \mathrm{~s}$ ) em repouso, além de uma menor variação na frequência cardíaca (FC) durante o período de sono dos animais do grupo experimental. Esses eventos não foram observados no grupo controle, o que sugere que o exercício físico extenuante interfere na modulação autonômica do coração, podendo predispor a arritmias importantes.
\end{abstract}

Palavras-chave: cão, exercícios físicos, Holter

\begin{abstract}
This study aimed to evaluate the effects of different loads of exercises on the heart rate behavior and rhythm in healthy dogs, over the 24-hour Holter recording. Twenty-three dogs were used without gender, age or breed restriction, and 15 animals were submitted to physical activities and 8 were used as control group. The results showed changes in heart rate during the proposed activities, with $174 \pm 27$ bpm while

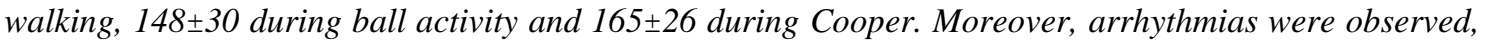
including atrioventricular blocks and premature complexes and a larger number and duration of pauses (up to $5 \mathrm{sec}$ ) at rest, and provided a smaller variation in the HR during the sleep period of animals from the experimental group. These events were not observed in the control group, suggesting that strenuous physical exercise affects the autonomic modulation of the heart, and may lead to important arrhythmias.
\end{abstract}

Keywords: dog; exercise; Holter

\section{INTRODUÇÃO}

A eletrocardiografia contínua, ou sistema Holter, constitui um método não invasivo de monitoração cardíaca, utilizado na prática clínica para detectar, documentar e caracterizar ocorrências de comportamentos anormais da atividade elétrica cardíaca durante as atividades diárias rotineiras (Grupi et al., 1999).

Recebido em 17 de março de 2012

Aceito em 13 de março de 2013

E-mail: faubarretto@gmail.com
Em medicina veterinária, o Holter está padronizado e vem sendo aprimorado por meio da avaliação de cães saudáveis e, principalmente, do estudo das cardiomiopatias (Ulloa et al., 1995; Kittleson e Kienle, 1998; Leomil Neto et al., 2002; Nogueira et al., 2006; NoszczykNowak et al., 2009). Em pequenos animais, essa ferramenta tem sido indicada para o diagnóstico de arritmias não sustentadas em pacientes com síncope, isquemia do miocárdio, cardiomiopatias (inclusive diagnóstico precoce), como a 
cardiomiopatia dilatada em cães e intolerância a exercícios (Kittleson e Kienle, 1998; Leomil Neto et al., 2002; Nogueira et al., 2006; Mucha e Belerenian, 2008; Oliveira et al., 2012). Outras indicações para essa técnica incluem a avaliação da necessidade de terapia antiarrítmica e sua eficácia (Petrie, 2005) e a avaliação da modulação autonômica do coração, por meio da análise da variabilidade da frequência cardíaca (Barretto et al., 2010; Barretto et al., 2011; Oliveira et al., 2012).

Em alguns casos, as variações no ritmo cardíaco podem ocorrer raramente, assim o eletrocardiograma deve ser registrado por longos períodos de tempo, sobretudo quando, nesse período de monitoramento, submete-se o paciente a uma condição de estresse controlado (Grupi et al., 2000). Embora a eletrocardiografia seja considerada o padrão ouro para detecção das arritmias (Tilley, 1992), neste caso a gravação Holter assume um papel essencial nas situações em que não se consegue submeter o animal à eletrocardiografia convencional, como durante o sono ou exercícios físicos (Calvert et al., 2000). Tais exercícios provocam importantes modificações no funcionamento do sistema cardiovascular e em seus mecanismos de modulação autonômica. Assim, o monitoramento cardíaco durante as atividades físicas torna-se relevante, permitindo uma análise adicional e não invasiva do controle neural da frequência cardíaca (Alonso et al., 1998).

Segundo a literatura, a prática de exercício físico regular, em longo prazo, promove melhora do aparato respiratório, endócrino, muscular, humoral e cardiovascular, sobretudo pela redução da pressão arterial sistêmica e por efeitos autonômicos antiarrítmicos, além de proporcionar melhora na qualidade do sono (Billman, 2002; Buch et al., 2002; Antunes et al., 2008). Tanto em seres humanos como em animais, é relatado que a atividade física atua na modulação do sistema nervoso autônomo, aumentando a atividade parassimpática ou reduzindo a simpática, principalmente no início do repouso (Billman, 2002; Buch et al., 2002; Martinelli et al., 2005; Guyton e Hall, 2006; Cavalcanti et al., 2009). Desta forma, um melhor conhecimento do padrão de sono frente ao exercício pode ser uma ferramenta importante para se alcançar maior desempenho físico, bem como para inferir o nível de estresse do treinamento, uma vez que alterações nesses padrões podem decorrer de um treinamento físico excessivo (Martins et al., 2001; Antunes et al., 2008).

Ainda, de acordo com Veerman et al. (1995), Piccione et al. (2005) e Oleskovicz et al. (2007), o sistema Holter pode ser uma importante ferramenta no estudo do ritmo circadiano, o qual exerce regulação em diversos sistemas fisiológicos, sobretudo o sistema cardiovascular. Acredita-se que um maior entendimento desse ritmo e de suas variações cardiovasculares observadas ao longo do dia pode ajudar a elucidar a interação entre o sistema nervoso autônomo, o ritmo circadiano do cortisol e as fases do sono (REM e NREM) e do despertar presentes no ciclo sono-vigília (Yamada e Tokuriki, 2000; Fernandes, 2006; Corrêa, 2008; Drager e Krieger, 2009).

Destarte, o presente estudo teve como objetivo avaliar os efeitos de diferentes cargas de exercícios físicos sobre o comportamento da frequência e do ritmo cardíaco de cães saudáveis, ao longo das 24 horas de gravação Holter.

\section{MATERIAL E MÉTODOS}

O presente estudo foi aprovado pelo Comitê de Ética para o Uso de Animais, sob registro CEUA-UENF 084. Empregaram-se 23 cães hígidos, sem restrição de raça, sexo, porte ou peso. Os animais foram selecionados no setor de Clínica Médica e Cardiológica do Hospital Veterinário da UENF, mediante realização de exame físico, anamnese, hemograma, pesquisa de hemocitozoários, bioquímica sérica dos níveis de ureia, creatinina, AST, ALT e fosfatase alcalina, eletrocardiografia convencional e ecodopplercardiografia. Animais com quaisquer indicativos de doença prévia ou concorrente foram excluídos do experimento.

Para a realização do Holter, os animais foram divididos em dois grupos. Os animais do grupo experimental (GE) foram submetidos a diversas atividades físicas padronizadas e enumeradas de acordo com a Tabela 1, e os do grupo controle (GC) foram mantidos conforme rotina habitual e avaliados a cada hora, totalizando 14 horas de vigília. A avaliação dos parâmetros durante o período de sono também foi realizada a cada hora, totalizando 10 horas de sono. 


\section{Eletrocardiografia contínua...}

As variáveis estudadas foram: frequência cardíaca média e mínima, ritmo cardíaco predominante, pausas superiores a dois segundos de duração entre os batimentos cardíacos e o comportamento da frequência cardíaca durante todo o período de gravação.

Tabela 1. Enumeração e duração das atividades físicas propostas. Hospital Veterinário/UENF, 2010.

\begin{tabular}{cccccc} 
Atividades & $\mathrm{N}^{\mathrm{o}}$ & $\begin{array}{c}\text { Duração } \\
(\mathrm{min})\end{array}$ & Atividades & $\mathrm{N}^{\mathbf{o}}$ & $\begin{array}{c}\text { Duração } \\
(\mathrm{min})\end{array}$ \\
\hline Pós-implantação do & 1 & 10 & Repouso & 9 & 90 \\
Holter & & & & & \\
Caminhada leve & 2 & 30 & Cooper & 10 & 15 \\
Repouso & 3 & 90 & Repouso & 11 & 135 \\
Atividade com bola & 4 & 30 & Afago & 12 & 30 \\
Repouso & 5 & 60 & Repouso & 13 & 60 \\
Almoço & 6 & 15 & Jantar & 14 & 15 \\
Repouso & 7 & 105 & Repouso & 15 & 75 \\
Atividade com & 8 & 30 & Atividade de saltar & 16 & 15 \\
corda/pano & & & & & \\
\hline
\end{tabular}

Para a realização do exame foi utilizado o Sistema de Holter CardioLight $\left(\operatorname{Cardios}^{\circledR}\right)$. Os eletrodos (Eletrodo para monitoração cardíaca 2223-3M®, 3M, Brasil) foram fixados na pele do animal, na região torácica, mediante tricotomia prévia e limpeza da região com álcool $70 \%$ para retirada dos pelos e da oleosidade da pele. Os eletrodos foram posicionados segundo o padrão das derivações pré-cordiais, conforme empregado por Barretto et al. (2010): eletrodo branco em $\mathrm{rV} 2$, eletrodo vermelho em V2, eletrodo preto em V4 e o verde entre o vermelho e o preto. Os eletrodos foram fixados com fita adesiva e envoltos por ataduras circundadas ao tórax do animal. Na ausência de uma jaqueta especialmente confeccionada para esse fim, o gravador Cardiolight (Cardios $®$, Brasil) foi acondicionado sobre um colete ajustável e fixado com auxílio de fita adesiva, como ilustrado na Figura 1.

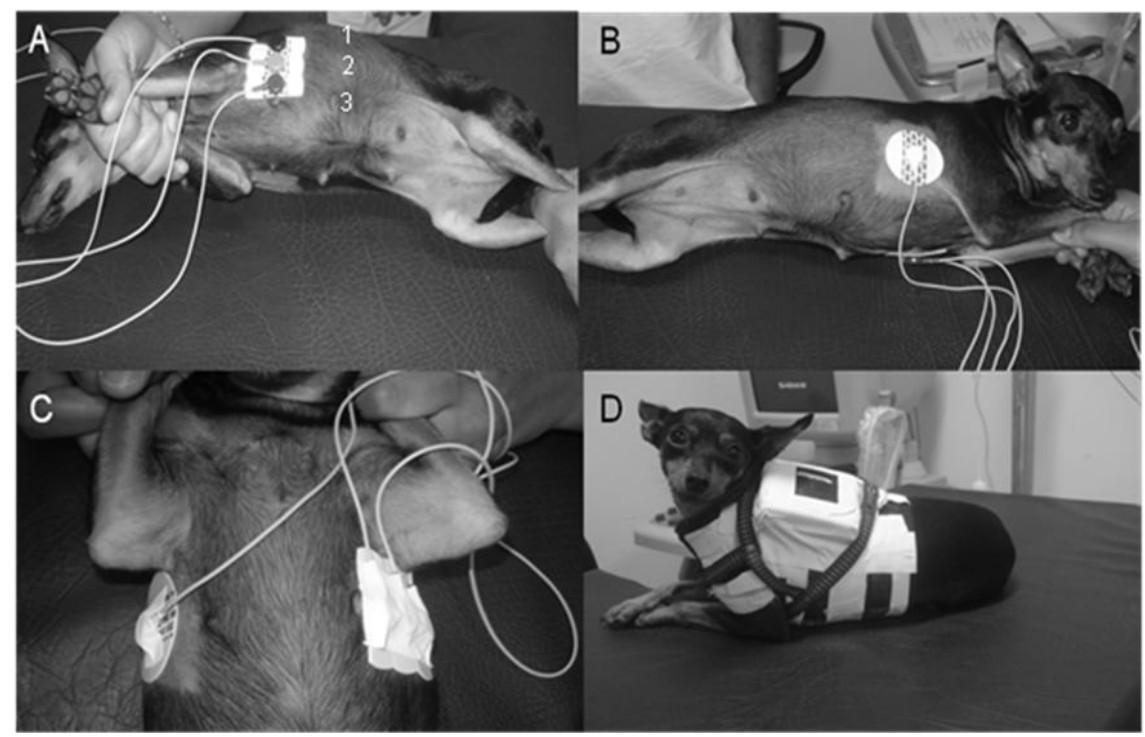

Figura 1. Implantação do sistema Holter em cão. Demonstrativo da fixação dos eletrodos e do gravador. Em A: 1 - eletrodo preto em V4; 2 - eletrodo verde entre V2 e V4; 3 - eletrodo vermelho em V2. Em B: eletrodo branco em rV2. Em C: fixação dos eletrodos com esparadrapo. Em D: acondicionamento do gravador sobre o colete e fixação com esparadrapo. Adaptado de Barretto et al., 2010. 
O registro contínuo foi realizado durante 24 horas na casa do proprietário, o qual ficou responsável por realizar as atividades com o seu cão e anotá-las, tais como sono, refeição, momentos de exaltação ou agressividade e outros aspectos que julgasse importante. $\mathrm{O}$ início do monitoramento foi padronizado para as nove horas da manhã.

A análise dos dados do Holter registrados no cartão de memória do gravador foi realizada por meio do software CardioManager 530 (Cardios $®$, Brasil). Os resultados observados para as variáveis em estudo foram tabulados e analisados com programa estatístico GraphPad Prism versão 4.03 para Windows (GraphPad Software, EUA) mediante o teste One-way ANOVA e o pós-teste de comparações múltiplas de Tukey, com significância estatística quando $p<0,05$. Ainda, foram calculados os valores de média, desvio-padrão e regressão linear.

\section{RESULTADOS E DISCUSSÃO}

A descrição da composição do GC e do GE, assim como os valores dos dados gerais obtidos na gravação do Holter, tais como: frequência cardíaca mínima (FC Mín) e média (FC Méd), percentual de artefato ( $\%$ artefato) e número total de batimentos cardíacos para as 24 horas de gravação ( $\mathrm{N}^{\circ}$ Total bpm), com seus respectivos valores médios e desvios-padrão, encontram-se na Tabela 2. A FC máxima não foi passível de análise, visto que o programa de análise de Holter utilizado é de uso humano e não precisa valores superiores a 250 batimentos por minuto (bpm).

Tabela 2. Identificação dos animais dos grupos controle e experimental, segundo sexo, idade, peso corpóreo, frequência cardíaca mínima e média, total de batimentos por minuto (bpm) e percentual de artefatos. F: Fêmea; M: Macho; DP: Desvio-padrão. Hospital Veterinário/UENF, 2010.

\begin{tabular}{ccccccccc} 
Animal & \multicolumn{2}{c}{ Sexo } & $\begin{array}{c}\text { Idade } \\
(\text { meses })\end{array}$ & $\begin{array}{c}\text { Peso } \\
(\mathrm{kg})\end{array}$ & $\begin{array}{c}\text { FC Mín } \\
(\mathrm{bpm})\end{array}$ & $\begin{array}{c}\text { FC Méd } \\
(\mathrm{bpm})\end{array}$ & $\begin{array}{c}\mathrm{N}^{\circ} \text { Total } \\
\text { bpm }\end{array}$ & $\begin{array}{c}\% \\
\text { artefatos }\end{array}$ \\
\hline GC & $5 \mathrm{~F}$ & $3 \mathrm{M}$ & $66 \pm 46$ & $9 \pm 7$ & $38 \pm 3$ & $79 \pm 11$ & $105762 \pm 11225$ & $3 \pm 3$ \\
GE & $11 \mathrm{~F}$ & $4 \mathrm{M}$ & $41 \pm 28$ & $9 \pm 10$ & $38 \pm 6$ & $94 \pm 12$ & $125149 \pm 15062$ & $5 \pm 5$ \\
\hline
\end{tabular}

O GC constituiu-se de quatro cães SRD (sem raça definida), dois Poodle, um Teckel e um Lhasa Apso, e o GE constituiu-se de quatro cães SRD, um Rottweiler, um Bull Terrier, um Yorkshire Terrier, um Schnauzer Miniatura, três Pinscher Miniatura, dois Maltês e um Teckel.

Mediante a análise da Tabela 2, é possível observar que o valor médio da FC Mín de ambos os grupos foi de $38 \mathrm{bpm}$, valor este considerado abaixo do estabelecido como normal, ou bradicardia $(<70 \mathrm{bpm})$, para cães em repouso (Tilley, 1992). Porém, os valores da frequência cardíaca mínima dos animais do presente estudo foram obtidos durante a madrugada, compreendendo o período de sono, quando há predominância do controle vagal sobre o coração (Noszczyk-Nowak et al., 2009).

Os valores médios da FC Méd obtidos no GC (79bpm) e GE (94bpm) encontram-se na mesma faixa que os observados por Yamada e Tokuriki (2000), Nogueira et al. (2006), Noszczyk-Nowak et al. (2009). Entretanto, a diferença observada entre esses grupos pode ser atribuída ao maior desgaste físico apresentado pelos animais do GE, visto que, quando o organismo é submetido a uma carga de esforço físico, a demanda de oxigênio e de nutrientes para os músculos aumenta, o que ativa mecanismos de regulação que promovem maior ativação do sistema simpático, aumentando a frequência de despolarização do nodo sinusal e, consequentemente, a frequência cardíaca (Alonso et al., 1998; Guyton e Hall, 2006). O mesmo se aplica ao número total de batimentos cardíacos obtidos nas 24 horas de gravação $\left(\mathrm{N}^{\circ}\right.$ Total bpm), uma vez que a média do GE (125.149bpm) foi superior à observada no GC (105.762bpm), como era esperado.

A média de percentual de artefatos ( $\%$ artefatos) do GE (5\%) se apresentou um pouco maior do que a observada no GC (3\%), o que pode ser atribuído à maior movimentação do GE, causando maior interferência na gravação. Contudo, em ambos os grupos, a quantidade de artefatos obtida nas gravações foi baixa e não prejudicou a análise dos exames. 
O ritmo cardíaco se comportou de forma padrão entre os animais de cada grupo. Os animais do GE apresentaram taquicardia sinusal nos momentos de exercício; nos demais momentos apresentaram ritmo sinusal ou arritmia sinusal respiratória, bradicardia e episódios de parada sinusal nos momentos de sono, assim como observado no GC.

A arritmia sinusal respiratória é bastante pronunciada em cães saudáveis, e em períodos de repouso, o incremento do sistema parassimpático pode resultar em bradicardia, ou mesmo em parada sinusal (Tilley, 1992). A taquicardia sinusal ocorre como resposta fisiológica ao medo, dor, estresse ou exercícios, para atender o aumento da demanda de oxigênio do organismo (Guyton e Hall, 2006; Mucha e Belerenian, 2008).

As arritmias cardíacas foram mais observadas nos animais do GE, sendo a de maior prevalência as extrassístoles ventriculares isoladas (ESV). Dois animais do GC apresentaram apenas um episódio de ESV observado no período de sono. Três animais do GE apresentaram um episódio de ESV, que ocorreu durante a noite em dois animais e durante uma atividade física em outro. Outros dois animais do GE apresentaram 280 e 91 episódios, respectivamente, sendo a maioria observada nos períodos de repouso após atividades físicas. Extrassístoles atriais (ESA) foram observadas em apenas dois animais do GE, tendo um apresentado 18 episódios durante uma atividade física e o outro 80 episódios em períodos de repouso. A incidência de bloqueio atrioventricular de $2^{\circ}$ grau (BAV $2^{\circ}$ grau) foi observada em apenas um animal do GC, que apresentou 12 episódios no decorrer da tarde e à noite, e em dois animais do GE, um apresentou apenas um episódio à noite, e o outro nove episódios em períodos de repouso. Um animal do GE apresentou bigeminismo ventricular no período de sono.

Períodos de BAV de $2^{\circ}$ grau podem ser observados em animais sadios em repouso, e episódios de ESV e ESA podem ser observados normalmente em alguns indivíduos, sendo que a prevalência e a frequência dessas arritmias aumentam com o avançar da idade (Petrie, 2005). Ulloa et al. (1995) avaliaram exames de Holter em cães saudáveis e diagnosticaram BAV de $2^{\circ}$ grau em $14 \%$ dos animais e ESV em $26 \%$ dos animais estudados, sendo que em alguns animais a ocorrência foi maior do que 10 episódios por hora. Noszczyk-Nowak et al. (2009) avaliaram 51 cães hígidos, entre os quais 13 animais apresentaram tais arritmias, tendo alguns animais apresentado mais de 1.300 episódios de ESA e 90 episódios de ESV. Resultados semelhantes aos aqui reportados também foram encontrados por Nogueria et al. (2006), ao avaliarem cães da raça Boxer saudáveis. Os autores relataram a prevalência de ESV, seguidas por alguns episódios de bigeminismo ventricular, ESA e trigeminismo ventricular.

O comportamento da frequência cardíaca observado no GC, no decorrer das 14 horas observadas durante a vigília, encontra-se ilustrado na Figura 2.

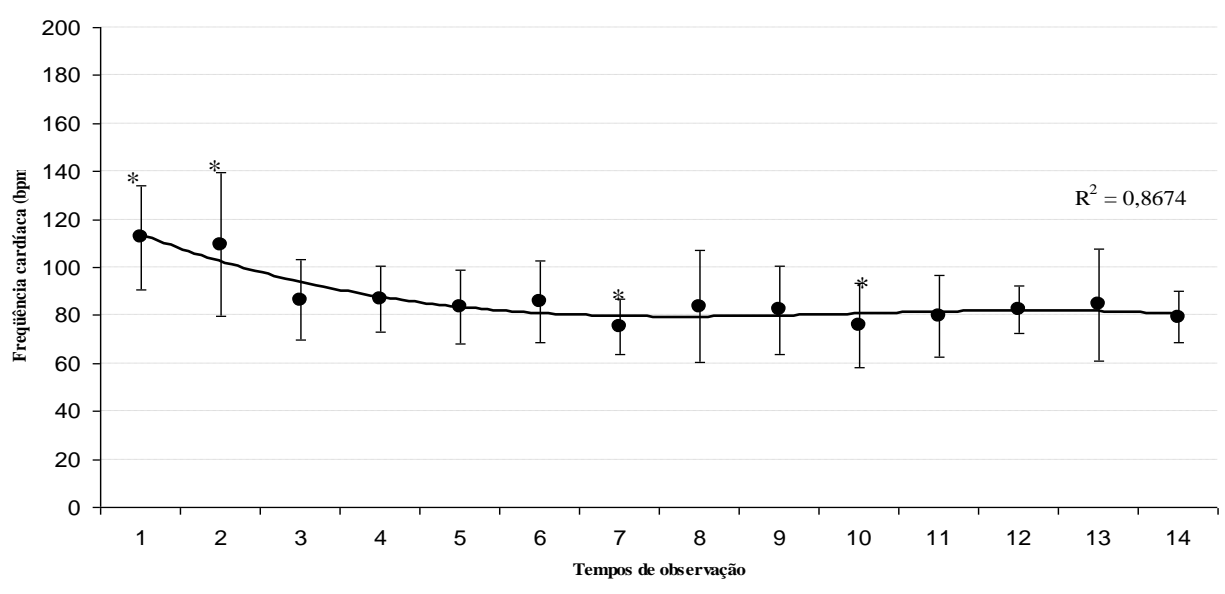

Figura 2. Comportamento da frequência cardíaca dos cães do grupo controle durante o período de vigília. Hospital Veterinário/UENF, 2010. *Análise estatística ( $\mathrm{p}<0,05)$; ANOVA e Tukey significativo. 
Mediante a análise da Figura 2, é possível observar que houve variação da frequência cardíaca (FC) do GC ao longo do dia, com diferença estatisticamente significativa entre as médias da FC ao longo do dia, comprovada pela análise de variância (ANOVA), com pós-teste Tukey, com $\mathrm{p}<0,05$.

Dentre as horas avaliadas destacam-se os pontos 1 e 2 , onde se observam as médias mais elevadas da FC (112 \pm 22 e $109 \pm 30$, respectivamente), além dos pontos 7 e 10, que apresentaram as menores médias ( $75 \pm 12$ e $76 \pm 18$, respectivamente). Os pontos 1 e 2 representam o período entre nove e 11 horas, correspondente ao período após a implantação do Holter, o que pode ter causado um aumento na FC devido ao estresse ao qual o animal foi submetido pela manipulação durante a implantação do aparelho. Já os pontos 7 e 10 representam os períodos das 15 às 16 , e das 18 às
19 horas, respectivamente. Sugere-se que estas médias mais baixas durante o dia sejam atribuídas ao período após a refeição, no caso do ponto 7, devido à maior estimulação parassimpática pós-prandial (Fernandes, 2006) e a períodos de repouso relatados na ficha de acompanhamento dos animais. Entretanto, apesar de ter havido diferença estatisticamente significativa entre os pontos demonstrados na Figura 2, houve pouca variação do comportamento da FC durante o dia, como pode ser observado na curva de tendência mais homogênea, comprovada pelo valor mais elevado do índice de aderência $\mathrm{R}^{2} \quad(0,8674)$, possibilitando concluir que não houve grande carga de estresse neste grupo.

O comportamento da frequência cardíaca do GE no decorrer das 16 atividades padronizadas ao longo do dia pode ser observado na Figura 3.

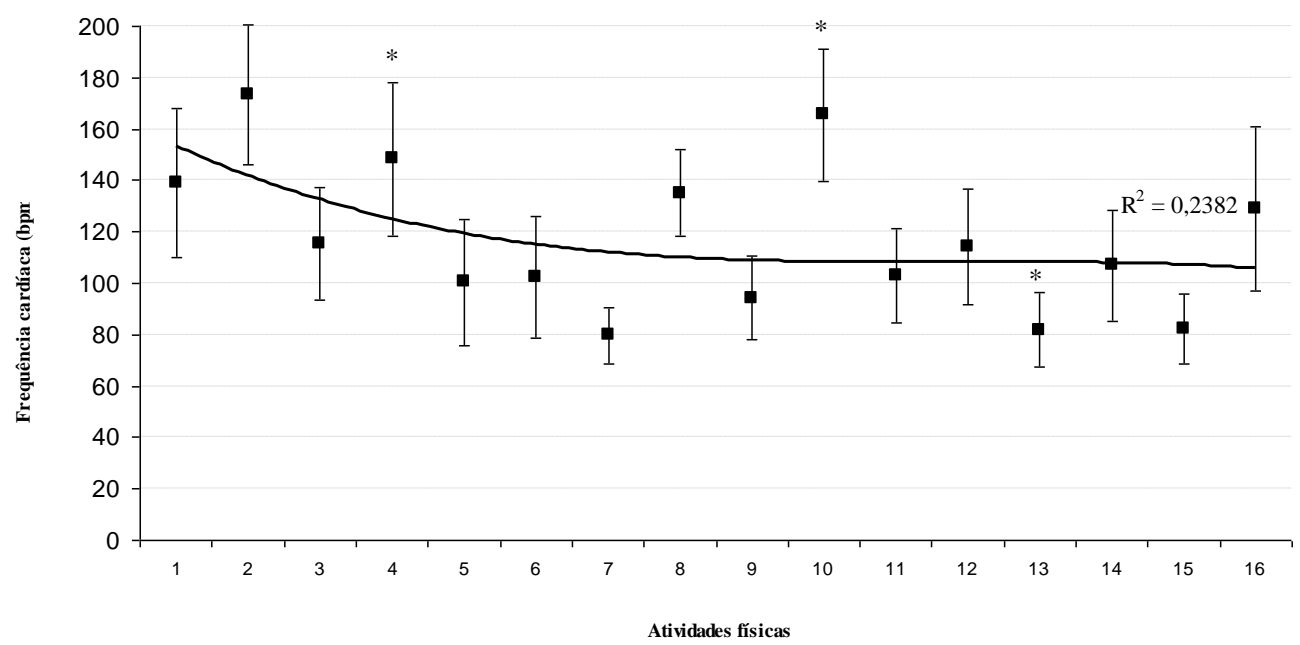

Figura 3. Comportamento da frequência cardíaca dos cães do grupo experimental durante as atividades físicas propostas. Legenda: 1 = pós-implantação do Holter; $2=$ caminhada leve; $3=$ repouso pós-caminhada leve; 4= atividade com bola; 5= repouso pós-atividade com bola; $6=$ almoço; $7=$ repouso pós-almoço; 8= atividade com corda ou pano; $9=$ repouso pós-atividade com corda ou pano; $10=$ Cooper $; 11=$ repouso pós-Cooper; 12= afago; 13= repouso pós-afago; 14= jantar; 15= repouso pós-jantar; 16= atividade de saltar. Hospital Veterinário/UENF, 2010. *Análise estatística $(\mathrm{p}<0,05)$; ANOVA e Tukey significativo.

Conforme evidenciado na Figura 3, nota-se que há diferença estatisticamente significativa com $\mathrm{p}<0,05$ entre as médias da FC observadas ao longo do período de atividades preestabelecidas para o GE. Pode-se também perceber que as FCs mais elevadas foram obtidas nos pontos 2 $(174 \pm 27), \quad 4 \quad(148 \pm 30)$ e $10 \quad(165 \pm 26)$, correspondentes a períodos de atividade física como caminhada leve, atividade com bola e Cooper, respectivamente. É importante ressaltar que, nas atividades em que a $\mathrm{FC}$ mostrou-se mais elevada (2, 4 e 10), não houve diferença estatística entre si.

Em oposição, ocorreu comportamento similar da FC nos pontos 7, 13 e 15, que representam períodos de repouso e onde foram registradas as menores médias da FC $(80 \pm 11,82 \pm 15$ e $82 \pm 14$, respectivamente), porém esses valores não demonstraram diferença estatisticamente 
significativa em relação à média da FC média (94bpm), indicando que o tempo estipulado para o repouso não foi suficiente para a recuperação da FC dos animais após o término da atividade anterior.

Diferentemente do observado no GC, por tratarse de um grupo em que as atividades foram estritamente controladas e com níveis intensos de atividades físicas, percebe-se, por meio da análise apresentada na Figura 3 , que o GE apresentou FCs mais heterogêneas, fato comprovado pelo índice de aderência $\mathrm{R}^{2}$ de 0,2382 contra o de 0,8674 observado no GC (Figura 2).

É possível observar, no entanto, que a FC apresentou uma tendência de redução no decorrer do dia em ambos os grupos, como demonstrado nas linhas de tendência das Fig. 2 e 3. Tal comportamento pode ser atribuído à regulação exercida pelo ritmo circadiano sobre o sistema cardiovascular. Os parâmetros cardiovasculares, tais como frequência cardíaca e pressão arterial, mostram valores mais altos durante o dia e mais baixos à noite. Essa variação resulta de estímulos externos e mecanismos endógenos de controle homeostático, como a observada no sistema autônomo, com incremento da atividade simpática durante o dia e redução à noite, ocorrendo o contrário com a atividade parassimpática em espécies de hábito diurno, assim como o oposto é observado em espécies de hábito noturno (Veerman et al., 1995; Piccione et al., 2005; Oleskovicz et al., 2007).

A quantidade de pausas com duração maior ou igual a dois segundos observadas no GC e GE encontra-se agrupada por tempo de duração, conforme mostra a Tabela 3. Também é mostrado o tempo de duração da maior pausa de cada grupo.

Ambos os grupos apresentaram maior percentual de pausas entre dois e três segundos $(97,99 \%)$. Porém, diferentemente do GC, o GE apresentou algumas pausas de duração mais elevada, sendo a maior delas com duração de cinco segundos. Outra diferença observada refere-se à quantidade total de pausas avaliadas, que no GE foi de 3.131 , valor superior ao registrado no GC.

Sabe-se que, durante períodos de repouso, a predominância do estímulo vagal pode resultar em grandes pausas entre os batimentos cardíacos, o que caracteriza a parada sinusal (sinus arrest), observada principalmente durante o sono (Tilley, 1992). Em cães saudáveis é relatado que pausas superiores a cinco segundos podem ser observadas em períodos de sono (Petrie, 2005). Entretanto, devido ao fato de a prática de exercícios físicos gerar aumento da atividade parassimpática no período pós-exercício (Billman, 2002; Buch et al., 2002), a maior ocorrência e duração de pausas observadas no GE poderiam ser justificadas pelo incremento vagal tardio, gerado pelo exercício.

Tabela 3. Distribuição das pausas, segundo duração, observadas nos cães dos grupos controle e experimental durante 24 horas de gravação Holter. DP: Desvio-padrão. Hospital Veterinário/UENF, 2010.

\begin{tabular}{ccccccccc}
\hline & \multicolumn{9}{c}{ Grupo experimental } & \multicolumn{5}{c}{ Grupo controle } \\
Pausas & $2-3 \mathrm{seg}$ & $3-4 \mathrm{seg}$ & $4-5 \mathrm{seg}$ & $5-6 \mathrm{seg}$ & $2-3 \mathrm{seg}$ & $3-4 \mathrm{seg}$ & $4-5 \mathrm{seg}$ & $5-6 \mathrm{seg}$ \\
\hline Subtotal & 3068 & 59 & 3 & 1 & 605 & 6 & - & - \\
Total & & & 3.131 & & & & 611 & \\
$\%$ & 97,99 & 1,88 & 0,1 & 0,03 & 99 & 1 & - & - \\
Média \pm DP & $205 \pm 316$ & $4 \pm 8$ & $0,2 \pm 0,8$ & $0,07 \pm 0,26$ & $76 \pm 93$ & $0,75 \pm 1$ & - & - \\
Maior pausa (seg) & - & - & - & 5 & - & 3,3 & - & - \\
\hline
\end{tabular}

A Figura 4 apresenta o comportamento da FC durante o período de sono dos cães do GC e do GE. Os valores mais baixos da FC observados no GC ocorreram entre as duas e cinco horas da manhã (tempos 4, 5 e 6). As respectivas frequências foram de $65 \pm 7,65 \pm 8$ e $65 \pm 6 b p m$. Já os valores mais baixos da FC do GE ocorreram entre cinco e oito horas da manhã (tempos 7,8 e
9). As respectivas frequências foram de $68 \pm 14$, $67 \pm 13$ e $68 \pm 13 \mathrm{bpm}$.

Ainda, pode-se observar que as médias da FC dos animais do GE decresceram continuamente até o momento 8 (entre seis e sete horas da manhã), com discreta elevação após este tempo. Os animais do GC apresentaram discretas 
oscilações na FC até o tempo 7 (entre cinco e seis horas da manhã), quando, a partir de então, as FCs se elevaram.

O aumento da FC pela manhã, observado em ambos os grupos, deve estar relacionado com o aumento da atividade simpática verificada na última fase do sono REM, que corresponde à fase imediata ao despertar (Drager e Krieger, 2009). O aumento da luminosidade pela manhã pode estar relacionado com o aumento da temperatura corporal interna, que é observado por volta das seis horas da manhã e facilita o despertar e a manutenção da vigíla (Yamada e Tokuriki, 2000; Fernandes, 2006). Tais achados também podem estar relacionados ao ritmo circadiano do cortisol, que é observado em espécias diurnas, apresentando pico pela manhã e declínio ao anoitecer (Corrêa, 2008).

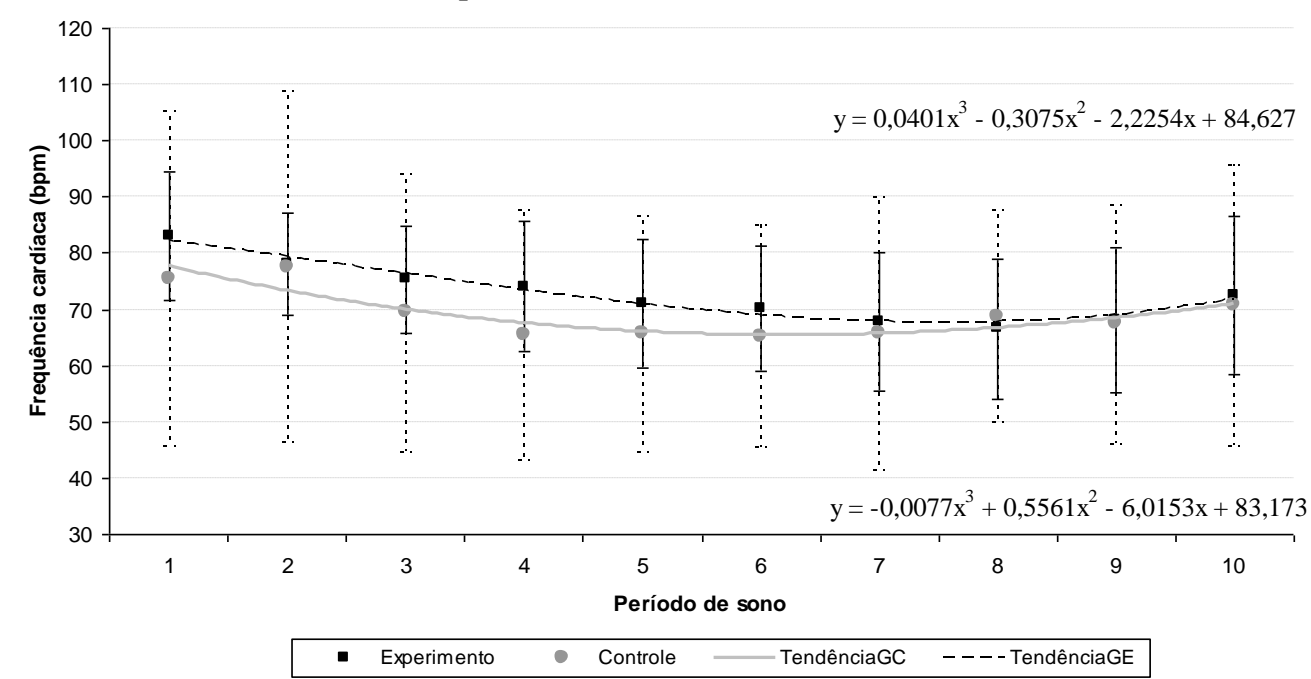

Figura 4. Comportamento da frequência cardíaca dos cães dos grupos controle e experimental durante o período de sono. Hospital Veterinário/UENF, 2010.

A diferença de horário encontrada no período precedente ao despertar, com incremento da FC, entre o GE (a partir das sete horas da manhã) e o GC (a partir das seis horas da manhã), pode ser atribuída à realização de exercícios físicos pelo GE, visto que o aumento do período total de sono pode estar associado à prática de exercícios físicos (Martins et al., 2001).

$\mathrm{Na}$ Figura 4, estão demonstradas as curvas de tendência (taxa de recuperação) da FC obtidas no período da noite do GC e do GE, em que se pode avaliar melhor o comportamento apresentado pela FC durante o sono. Em virtude da realização das atividades físicas, o GE atingiu o sono mais rapidamente do que o $\mathrm{GC}$, o que pode ser comprovado pelas equações de tendência apresentadas no gráfico.

O declínio mais rápido da FC observado no GE pode ser atribuído ao aumento da atividade parassimpática gerada pela prática de exercícios físicos (Billman, 2002; Buch et al., 2002). O incremento do tônus vagal, associado ao aumento da temperatura corporal interna e ao aumento do gasto energético, devido à alta atividade catabólica do exercício, pode ter levado à redução do período de latência para o sono, além de ter favorecido a ocorrência de um sono mais profundo, com predomínio do sono NREM, como tem sido descrito em humanos (Martins et al., 2001; Fernandes, 2006). Esse fato não foi observado no GC, que apresentou oscilações mais evidentes da frequência cardíaca, fato que pode estar relacionado ao menor tônus parassimpático neste grupo quando comparado ao grupo experimental.

Cavalcanti et al. (2009) encontraram resultados semelhantes ao compararem o comportamento da FC de cães praticantes de atividades físicas regulares com cães sedentários: os primeiros demonstraram redução da FC mais rapidamente ao longo do dia, sendo verificada com maior evidência após as 22 horas.

Estes resultados indicam que a diminuição da frequência cardíaca dos atletas se deve 
ao aumento da influência parassimpática, com diminuição do ritmo intrínseco do nodo sinoatrial, estando este possivelmente condicionado à quantidade de acetilcolina encontrada no tecido auricular depois do exercício e a uma diminuição da sensibilidade do tecido cardíaco às catecolaminas (Buch et al., 2002; Martinelli et al., 2005).

\section{CONCLUSÕES}

Pelo presente estudo, conclui-se que: a- a eletrocardiografia contínua (Holter) constitui uma técnica viável para a avaliação da frequência cardíaca e do ritmo cardíaco em cães clinicamente sadios submetidos a diferentes cargas de exercício físico; b- a prática das atividades físicas propostas predispõe à maior ocorrência de arritmias cardíacas e de pausas durante o período de sono, induz ao sono mais rapidamente, além de aumentar o período de sono total; c- a prática de exercícios físicos inadvertidamente pode predispor a arritmias importantes. Nesse âmbito, mais estudos devem ser realizados, a fim de se obter um melhor esclarecimento desses efeitos.

\section{REFERÊNCIAS}

ALONSO, D.O.; FORJAZ, C.L.M.; REZENDE, L.O. et al. Comportamento da Frequência Cardíaca e da Sua Variabilidade Durante as Diferentes Fases do Exercício Físico Progressivo Máximo. Arq. Bras. Cardiol., v.71, p.787-792, 1998.

ANTUNES, H.K.M.; ANDERSEN, M.L.; TUFIK, S. et al. Privação de Sono e Exercício Físico. Rev. Bras. Med. Esporte, v.14, p.51-56, 2008.

BARRETTO, F.L.; FERREIRA, F.S.; CORREA, E.S. et al. Monitorización eletrocardiográfica continua (Holter) de perros saludables sometidos a diferentes actividades físicas: resultados perliminares. Selecciones Vet., v.18, p.14-20, 2010.

BARRETTO, F.L.; FERREIRA, F.S.; CORREA, E.S. et al. Eletrocardiografia contínua (Holter) em cães Revisão de Literatura. MEDVEP, v.9, p.558-565, 2011.

BILLMAN, G.E. Aerobic exercise conditioning: a nonpharmacological antiarrhythmic intervention. $J$. Appl. Physiol., v.92, p.446-454, 2002.

BUCH, N.A.; COOTE, J.H.; TOWNEND, J.N. Mortality, cardiac vagal control and physical trainingwhat`s the link? Exp. Physiol., v.87, p.423-435, 2002.
CALVERT, C.A.; JACOBS, G.; PICKUS, C.W. et al. Results of ambulatory electrocardiography in overtly healthy Doberman Pinschers with echocardiographic abnormalities. J. Am. Vet. Med. Assoc., v.217, p.13281332, 2000.

CAVALCANTI, G.A.O.; NOGUEIRA, R.B.; SAMPAIO, G.R. et al. Avaliação por eletrocardiografia contínua (holter) em cães da raça Pastor Alemão praticantes de atividade física regular. Arq. Bras. Med. Vet. Zootec., v.61, p.1446-1449, 2009.

CORRÊA, P.M. Teste de supressão pela dexametasona em cães (Canis familiaris) com distúrbios comportamentais. 2008. 39f. Dissertação (Mestrado em Medicina Veterinária) - Curso de PósGraduação em Medicina Veterinária, Universidade Federal Rural do Rio de Janeiro, Rio de Janeiro.

DRAGER, L.F.; KRIEGER, E.M. Mecanismos de controle da pressão arterial no sono. Rev. bras. hipertens., v.16, p.169-173, 2009.

FERNANDES, R.M.F. O sono normal. In: SIMPÓSIO: DISTÚRBIOS RESPIRATÓRIOS DO SONO, 39., 2006, Ribeirão Preto. Anais. São Paulo: 2006. p.157-168. (Capítulo I).

GRUPI, C.J.; BRITO, F.S.; UCHIDA, A.H. Eletrocardiograma de Longa Duração: o Sistema Holter - Parte I. Reblampa, v.12, p.86-92, 1999.

GRUPI, C.J.; BRITO, F.S.; UCHIDA, A.H. Eletrocardiograma de Longa Duração: o Sistema Holter - Parte III. Reblampa, v.13, p.71-76, 2000.

GUYTON, A.C.; HALL, J.E. Tratado de Fisiologia Médica. Rio de Janeiro: ELSEVIER, 2006. 1115p.

KITTLESON, M.D.; KIENLE, R.D. Small animal cardiovascular medicine. Baltimore: MOSBY, 1998. $603 \mathrm{p}$.

LEOMIL NETO, M.; LARSSON, M.H.M.A.; PEREIRA, L. et al. Padronização da monitorização eletrocardiográfica por 24 horas em cães. Arq. Bras. Med. Vet. Zootec., v.54, p.133-138, 2002.

MARTINELLI, F.S.; CHACON-MIKAHIL, M.P.T.; MARTINS, L.E.B. et al. Heart rate variability in athletes and nonathletes at rest and during head-up tilt. Braz. J. Med. Biol. Res., v.38, p.639-647, 2005.

MARTINS, P.J.F.; MELlO, M.T.; TUFIK, S. Exercício e sono. Rev. Bras. Med. Esporte, v.7, p.2836, 2001.

MUCHA C.J.; BELERENIAN G. Manual de Cardiologia Veterinária. Buenos Aires: HOLLIDAYSCOTT S.A., 2008. 112p.

NOGUEIRA, R.B.; MUZZI, R.A.L.; HERRERA, D.S. et al. Avaliação do ritmo cardíaco em cães da raça Boxer saudáveis pela eletrocardiografia contínua 
(Holter). Arq. Bras. Med. Vet. Zootec., v.58, p.133136, 2006.

NOSZCZYK-NOWAK, A.; PASLAWSKA, U.; NICPON, J. ECG parameters in 24-hour Holter monitoring in healthy dogs. Bull. Vet. Inst. Pulawy., v.53, p.499-502, 2009.

OLESKOVICZ, N.; DUQUE, J.C.; GUIRRO, E.C.P. et al. Avaliação através de Biotelemetria das Flutuações Diárias da Pressão Arterial e Frequência Cardíaca em cães da raça Beagle. ARS Veterinaria, v.23, p.134-140, 2007.

OLIVEIRA, M.S.; MUZZI, R.A.L.; ARAÚJO, R.B. et al. Heart rate variability parameters of myxomatous mitral valve disease in dogs with and without heart failure obtained using 24-hour Holter electrocardiography. Vet. Rec., v.170, p.622-625, 2012.

PETRIE, J.P. Practical application of Holter monitoring in dogs and cats. Clin. Tech. Small. Anim. Pract., v.20, p.173-181, 2005.
PICCIONE, G.; GRASSO, F.; GIUDICE, E. et al. Circadian rhythm in the cardiovascular system of domestic animals. Res. Vet. Sci., v.79, p.155-160, 2005.

TILLEY, L.P. Essentials of canine and feline electrocardiography. 3.ed. Philadelphia: LEA \& FEBIGER, 1992. 470p.

ULLOA, H.M.; HOUSTON, B.J.; ALTROGGE, D.M. Arrhythmia prevalence during ambulatory electrocardiographic monitoring of Beagles. Am. J. Vet. Res., v.56, p.275-281, 1995.

VEERMAN, D.P.; IMHOLZ, B.P.M.; WIELING, W. et al. Circadian Profile of Systemic Hemodynamics. Hypertension, v.26, p.55-59, 1995.

YAMADA, M.; TOKURIKI, M. Effects of a Canine Elizabethan Collar on Ambulatory Electrocardiogram Recorded by a Holter Recording System and Spontaneous Activities Measured Continuously by an Accelerometer in Beagle Dogs. J. Vet. Med. Sci., v.62, p.549-552, 2000. 